Article

\title{
Red Blood Cell Docosapentaenoic Acid (DPA $n$-3) is Inversely Associated with Triglycerides and C-reactive Protein (CRP) in Healthy Adults and Dose-Dependently Increases Following $n-3$ Fatty Acid Supplementation
}

\author{
Ann C. Skulas-Ray ${ }^{1}{ }^{*}$, Michael R. Flock ${ }^{1}$, Chesney K. Richter ${ }^{1}$, William S. Harris ${ }^{2}$, \\ Sheila G. West ${ }^{1,3}$ and Penny M. Kris-Etherton ${ }^{1}$ \\ ${ }^{1}$ Department of Nutritional Sciences, The Pennsylvania State University, University Park, \\ PA 16802, USA; E-Mails: mike.flock@gmail.com (M.R.F.); ckr129@psu.edu (C.K.R.); \\ sgw2@psu.edu (S.G.W.); pmk3@psu.edu (P.M.K.-E.) \\ ${ }^{2}$ OmegaQuant Analytics, LLC, Sioux Falls, SD 57106, USA; E-Mail: Bill@ omegaquant.com \\ ${ }^{3}$ Department of Biobehavioral Health, The Pennsylvania State University, University Park, \\ PA 16802, USA \\ * Author to whom correspondence should be addressed; E-Mail: aus164@ psu.edu; \\ Tel.: +1-814-863-8622.
}

Received: 1 June 2015 / Accepted: 21 July 2015 / Published: 4 August 2015

\begin{abstract}
The role of the long-chain omega-3 (n-3) fatty acids eicosapentaenoic acid (EPA) and docosahexaenoic acid (DHA) in lipid metabolism and inflammation has been extensively studied; however, little is known about the relationship between docosapentaenoic acid (DPA, 22:5 n-3) and inflammation and triglycerides (TG). We evaluated whether $n$-3 DPA content of red blood cells (RBC) was associated with markers of inflammation (interleukin-6 (IL-6), tumor necrosis factor $\alpha$ (TNF- $\alpha$ ), and C-reactive protein (CRP) and fasting TG prior to $n-3$ supplementation in two studies (Study 1: $n=115$, aged 20-44 years, body mass index (BMI) 20-30 kg/m², TG = 34-176 mg/dL; Study 2: $n=28$, aged 22-65 years, BMI $\left.24-37 \mathrm{~kg} / \mathrm{m}^{2}, \mathrm{TG}=141-339 \mathrm{mg} / \mathrm{dL}\right)$. We also characterized the dose-response effects of $n$-3 fatty acid supplementation on RBC $n$-3 DPA after five months of supplementation with fish oil (Study 1: 0, 300, 600, 900, and $1800 \mathrm{mg} /$ day EPA + DHA) and eight weeks of prescription $n$-3 ethyl esters (Study 2: 0, 850, and $3400 \mathrm{mg} /$ day EPA + DHA). In Study 1, RBC $n$-3 DPA was inversely correlated with CRP $\left(R^{2}=36 \%, p<0.001\right)$ and with fasting TG $(r=-0.30, p=0.001)$. The latter finding was replicated in Study $2(r=-0.33, p=0.04)$. In both studies, $n$-3 supplementation significantly increased RBC $n$-3 DPA dose-dependently. Relative increases were greater for Study 1, with increases of $29 \%-61 \%$ vs. $14 \%-26 \%$ for
\end{abstract}


Study 2. The associations between RBC $n-3$ DPA, CRP, and fasting TG may have important implications for the prevention of atherosclerosis and chronic inflammatory diseases and warrant further study.

Keywords: fish oil; marine omega-3 fatty acids; DPA; inflammation

\section{Introduction}

Long-chain omega-3 (n-3) polyunsaturated fatty acids (PUFA) reduce blood triglycerides [1,2] and play a role in preventing inflammatory diseases [3]. The relationships between intake and/or biomarkers of eicosapentaenoic acid (EPA, 20:5 n-3) and docosahexaenoic acid (DHA, 22:6n-3) and blood concentrations of triglycerides and inflammatory markers have been studied extensively $[4,5]$. However, limited research has examined associations between these risk factors and docosapentaenoic acid (DPA, 22:5n-3), which is also present in $n-3$ supplements in varying concentrations and is commonly consumed in seafood and red meat from ruminant animals [6].

Studies of plasma and serum n-3 DPA concentrations indicate that $n-3$ DPA also likely plays a role in influencing health outcomes that are responsive to EPA and DHA. Lower serum concentrations of DHA $+n-3$ DPA and n-3 DPA alone have been associated with greater risk of acute coronary events [7] and myocardial infarction [8], respectively. Plasma n-3 DPA was also inversely associated with total mortality (particularly from stroke-related deaths) [9], nonfatal myocardial infarction [10], and incident cardiovascular disease (CVD) in some ethnic groups [11]. Inverse associations have also been found between serum or plasma $n$-3 DPA and the C-reactive protein (CRP) [12,13].

Erythrocyte or red blood cell (RBC) fatty acid content may serve as a better biomarker than plasma for examining the relationship between $n-3$ DPA and disease risk factors. The fatty acid content of RBC membranes better reflects longer term dietary intakes than plasma and serum levels [14]. RBC membrane fatty acids also correlate with concentrations in other tissues/cells, and are associated with clinically relevant outcomes $[15,16]$. RBC fatty acids have been utilized extensively as a biomarker in studies of EPA and DHA [17], but few studies have reported relationships between RBC $n-3$ DPA and triglycerides or inflammatory markers despite the fact that $n-3$ DPA is present in RBC membranes in amounts comparable to EPA and DHA.

Little is known about how RBC $n-3$ DPA responds to $n-3$ supplementation. Limited evidence suggests that fish oil supplementation increases RBC $n$-3 DPA content [18]. Fish oil contains a relatively low concentration of DPA, and prior studies have not typically reported the DPA content of their supplements. Providing a complete characterization of the $n$ - 3 fatty acid profile of supplements would aid in the interpretation of RBC n-3 DPA responses. In addition to the n-3 DPA provided by supplements, endogenous production from other supplemented $n-3$ fatty acids, particularly EPA, may also be an important means of increasing tissue $n-3$ DPA reserves. For instance, plasma $n-3$ DPA increased significantly following supplementation with concentrated EPA ethyl esters [19,20] whereas no change in RBC n-3 DPA was found after three months of supplementation with a DHA-rich tuna oil [21]. A better understanding of how RBC $n$-3 DPA content responds to increased $n$ - 3 intake would provide insight needed to better characterize the role of $n-3$ DPA in the health effects attributed to $n-3$ fatty acids. 
If RBC $n-3$ DPA levels are associated with triglycerides and inflammatory markers, and are responsive to $n-3$ supplementation, it is feasible that $n-3$ DPA may, in part, be responsible for some of the biological effects attributed to EPA and DHA. Thus, these evaluations are needed to increase our understanding of the potentially important biological effects of $n-3$ DPA. In order to evaluate the relationship between RBC $n-3$ DPA concentrations, triglycerides, and inflammation, we utilized data from two $n-3$ supplementation studies that enrolled healthy young adults $(n=115)$ and individuals with elevated triglycerides $(n=28)$ to examine associations between pre-supplementation RBC $n-3$ DPA content and markers of inflammation (interleukin-6 (IL-6), tumor necrosis factor $\alpha$ (TNF- $\alpha$ ), and CRP and fasting triglycerides. We also evaluated the dose response effects of RBC n-3 DPA to $n-3$ supplementation in the two studies, which included different doses of fish oil (300, 600, 900, and $1800 \mathrm{mg} /$ day EPA + DHA) administered for approximately five months and a prescription $n-3$ fatty acid ethyl ester concentrate (LovazaTM, $850 \mathrm{mg} /$ day and $3400 \mathrm{mg} /$ day EPA + DHA) administered for eight weeks to inform understanding of how increased $n-3$ intake affects endogenous stores of $n-3$ DPA.

\section{Experimental Section}

\subsection{Experimental Overview}

Experimental methods are described in detail in the original study reports [22,23]. Both studies (Figure 1) were registered on the web ClinicalTrials.gov (NCT01078909 and NCT00504309), conducted at the Clinical Research Center at the Pennsylvania State University, and approved annually by the Pennsylvania State University Institutional Review Board.

(A) Study 1

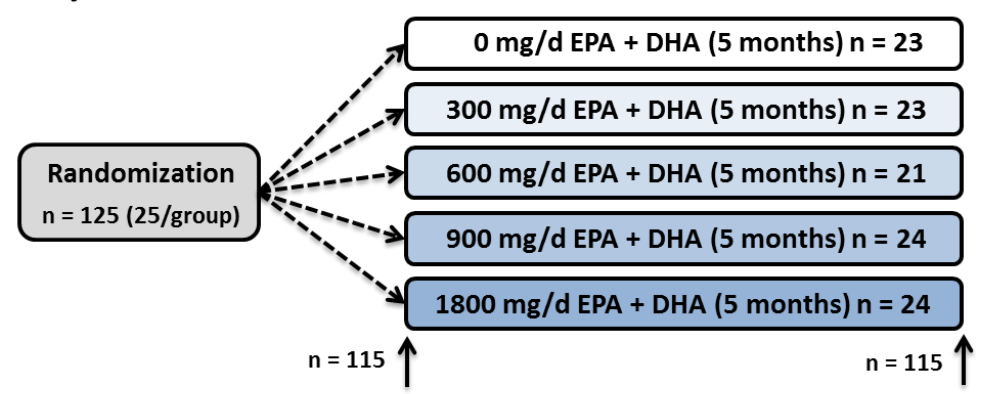

(B) Study 2

Period 1

$n=115 \uparrow$

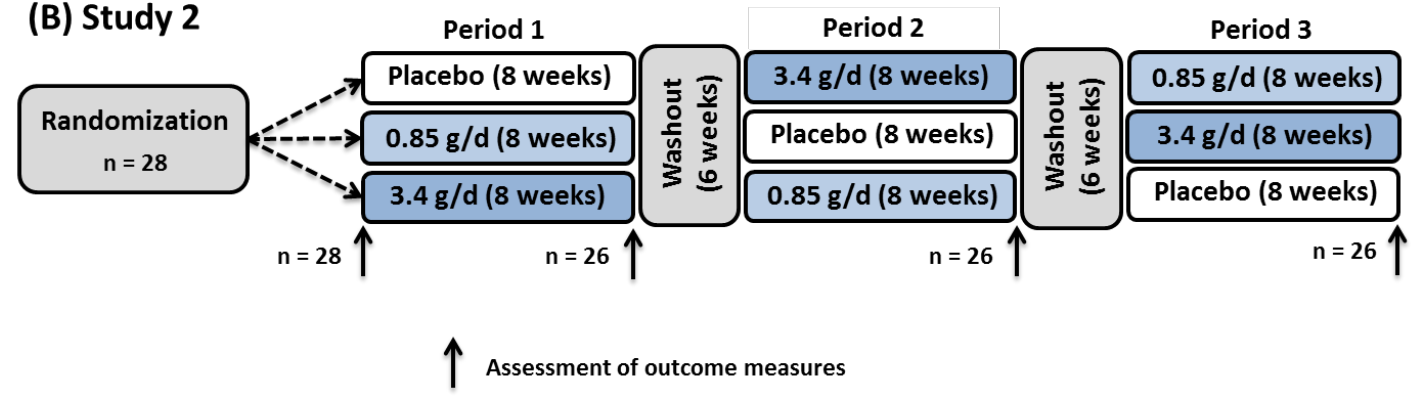

Figure 1. Study design schematic for Study 1 (A) and Study 2 (B). DHA, docosahexaenoic acid; EPA, eicosapentaenoic acid.

In Study 1 [22] (Figure 1A; Table 1), healthy young adults (20-45 years of age, body mass index (BMI) 20-30 kg/m², triglycerides (TG) 34-176 mg/dL) reporting no or low habitual oily fish 
consumption ( $<4$ meals per month) and not taking n-3 PUFA supplements were randomly assigned to one of five doses of daily fish oil supplements (0, 300, 600, 900, $1800 \mathrm{mg}$ EPA + DHA; soybean oil placebo) for approximately five months (Nordic Naturals, Watsonville, CA, USA). Of the 125 participants enrolled, 116 completed the study; however, one participant was excluded from analyses due to a baseline omega-3 index $>8 \%$ (indicating high $n-3$ intake).

Table 1. Study design and baseline characteristics of participants who completed Study 1 and Study $2^{1}$.

\begin{tabular}{ccc}
\hline & Study $\mathbf{1}$ & Study 2 \\
\hline Study design & Parallel & Crossover \\
Duration of supplementation & 20 weeks & 8 weeks \\
Total sample size & 115 & 26 \\
Male, $\boldsymbol{n}(\boldsymbol{\%})$ & $60(52 \%)$ & $23(88 \%)$ \\
Age $(\mathbf{y e a r})$ & $26.1 \pm 6.6(20-45)$ & $44.3 \pm 9.8(22-65)$ \\
BMI $\left(\mathbf{k g} / \mathbf{m}^{\mathbf{2}}\right)$ & $24.4 \pm 2.5(20-30)$ & $29.0 \pm 3.6(23.7-36.5)$ \\
Triglycerides $(\mathbf{m g} / \mathbf{d L})$ & $89.7 \pm 32.1(34-176)$ & $222.8 \pm 56.3(140.5-339)$ \\
CRP $(\mathbf{m g} / \mathbf{L})$ & $1.8 \pm(<0.2-28.8)$ & $1.3 \pm 0.8(<0.2-2.8)$ \\
RBC $\boldsymbol{n - 3}$ DPA content & $2.4 \% \pm 0.5(1.3 \%-3.6 \%)$ & $2.7 \% \pm 0.4(2.0 \%-3.5 \%)$ \\
\hline
\end{tabular}

1 Values are means \pm standard deviation (SD) with ranges in parentheses; BMI, body mass index; CRP, C-reactive protein; RBC, red blood cells.

In Study 2 [23] (Figure 1B; Table 1), individuals who had moderately elevated triglycerides (141-339 mg/dL) but were otherwise healthy (22-65 years of age, BMI $24-37 \mathrm{~kg} / \mathrm{m}^{2}$ ) and reported low habitual oily fish consumption ( $<2$ servings/week) were enrolled in a three-period randomized crossover study comparing the effects of 850 and $3400 \mathrm{mg} /$ day EPA + DHA to corn oil placebo. Each supplementation period lasted eight weeks and was separated by a six-week washout period. Twenty-eight participants were randomized to treatment groups and used for baseline analyses. Two participants did not complete the study, thus supplementation responses were assessed in 26 participants.

The long-chain $n-3$ fatty acid content provided by each supplement dose in Study 1 and Study 2, as determined by independent analysis, is provided in Table 2 [22,23].

Table 2. Omega-3 fatty acid content (mg/day) of each supplement dose in Study 1 and Study $2^{1}$.

\begin{tabular}{ccccccccccc}
\hline & \multicolumn{4}{c}{ Study $\mathbf{1}$} & \multicolumn{3}{c}{ Study 2 } \\
\cline { 2 - 10 } & \multicolumn{4}{c}{ Dose $(\mathbf{m g} / \mathbf{d a y})$} & \multicolumn{4}{c}{ Dose (mg/day) } \\
\hline Fatty acid & $\mathbf{0}$ & $\mathbf{3 0 0}$ & $\mathbf{6 0 0}$ & $\mathbf{9 0 0}$ & $\mathbf{1 8 0 0}$ & $\mathbf{0}$ & $\mathbf{8 5 0}$ & $\mathbf{3 4 0 0}$ \\
Eicosapentaenoic acid (EPA) & 9 & 191 & 374 & 556 & 1103 & 0 & 486 & 1944 \\
Docosapentaenoic acid (DPA $\boldsymbol{n - 3 )}$ & 1 & 20 & 40 & 59 & 118 & 0 & 35 & 141 \\
Docosahexaenoic acid (DHA) & 6 & 121 & 237 & 352 & 698 & 0 & 421 & 1686 \\
Total EPA + DHA & 15 & 312 & 611 & 908 & 1801 & 0 & 907 & 3630 \\
Total EPA + DPA + DHA & 16 & 332 & 651 & 967 & 1919 & 0 & 942 & 3771 \\
\hline
\end{tabular}

1 Values were calculated from independent analysis of fatty acid composition of a sample of active and placebo capsules. 


\subsection{Blood Sample Collection and Analysis}

Both studies utilized the same methods for blood sample collection, measurement of inflammatory markers, and analysis of RBC fatty acid (FA) composition. At baseline and following treatment periods, a venipuncture blood sample was obtained from participants following a 12-h overnight fast. Whole blood was centrifuged at $1500 \times g$ for $15 \mathrm{~min}$ at $4{ }^{\circ} \mathrm{C}$. Triglycerides were measured by enzymatic procedures (Quest Diagnostics, Pittsburgh, PA, USA; Coefficient of variation $(\mathrm{CV})<2 \%$ ). Aliquots of serum and plasma were collected and stored at $-80{ }^{\circ} \mathrm{C}$ until analysis. Serum concentrations of TNF- $\alpha$ and IL- 6 were measured using high-sensitivity enzyme-linked immunosorbent assay (ELISA) kits (R\&D Systems, Minneapolis, MN, USA) in duplicate $(\mathrm{CV}<10 \%)$. Serum high-sensitivity CRP was measured by latex-enhanced immunonephelometry (Quest Diagnostics; assay CV < 8\%). RBC were collected following separation from plasma by centrifugation and frozen at $-80{ }^{\circ} \mathrm{C}$ prior to analysis. As previously described, RBC FA composition was analyzed by gas chromatography with flame ionization detection [24]. Briefly, lipids were extracted, methylated to form fatty acid methyl esters (FAMEs), and analyzed by gas chromatography on a GC2010 (Shimadzu Corporation, Columbia, MD, USA) equipped with a 100-m SP-2560 column (Supelco, Bellefonte, PA, USA). Fatty acids were identified by comparison with a standard mixture of FAs characteristic of RBCs (GLC 727; Nu-Check Prep, Waterville, MN, UAS) that differentiated between $n-3$ and $n-6$ DPA isomers (Supplemental Figure S1). FAME composition was expressed as a percentage of total identified FAMEs $(\mathrm{CV}<3.7 \%)$.

\subsection{Statistical Analysis}

Regression analyses for pre-supplementation associations were performed using Minitab (version 17, Minitab, State College, PA, USA). The assistant function was utilized to select between linear, quadratic, or cubic models. Residual $v s$. fit plots were examined to ensure homoscedasticity. To avoid extreme outliers due to acute infection in Study 1, baseline CRP values exceeding $10 \mathrm{mg} / \mathrm{L}(n=3)$ were excluded from analysis. For Study 1, analysis of variance (ANOVA) was used to assess between group differences in RBC n-3 DPA responses. For Study 2, which was a crossover design, RBC $n$-3 DPA responses to supplementation were evaluated using the mixed models procedure (PROC MIXED) in SAS (Statistical Analysis System; version 9.3; SAS Institute Inc., Cary, NC, UAS). Subject was treated as a random effect, and the effects of treatment, period, and treatment by period interactions were assessed. Period and treatment by period interactions were non-significant and removed from the final model of treatment effects. Tukey-adjusted $p$-values were used for post hoc comparisons between treatment groups.

\section{Results}

\subsection{Pre-Supplementation RBC n-3 DPA: Associations with Fasting Triglycerides and Markers of} Inflammation, and Sex Differences

Baseline RBC $n$-3 DPA content and fasting triglycerides were inversely associated in both Study 1 (Figure 2A, $r=-0.30, p=0.001$ ) and Study 2 (Figure 2B, $r=-0.33, p=0.04$ ). For every $1 \%$ increase in baseline RBC $n-3$ DPA, triglycerides were decreased by $20 \mathrm{mg} / \mathrm{dL}$ in Study 1 and by $59 \mathrm{mg} / \mathrm{dL}$ in Study 2. In both studies, baseline fasting triglycerides were not significantly related to the RBC content of EPA, DHA, or the Omega-3 Index (EPA + DHA, data not shown). 
(A) Study 1

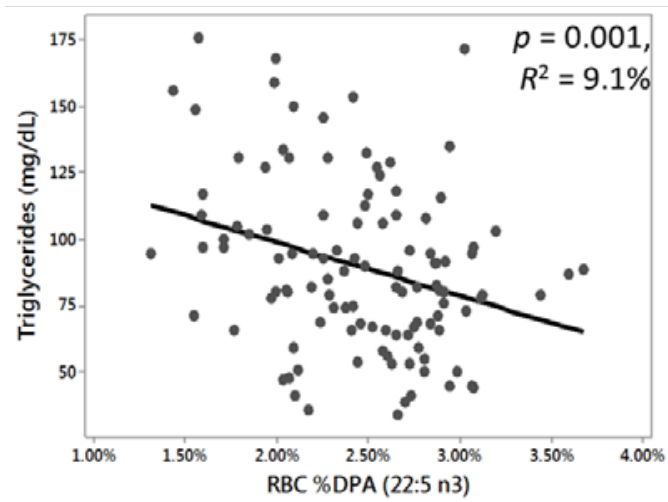

(B) Study 2

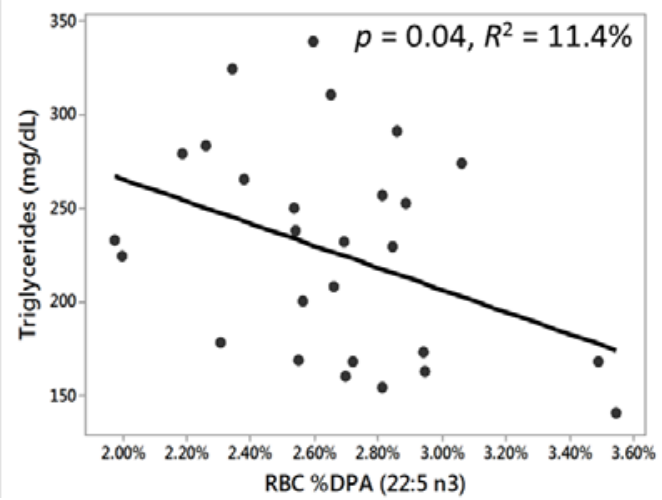

Figure 2. Scatterplots for regression analyses of serum triglycerides vs. red blood cell (RBC) \% docosapentaenoic acid (DPA $n-3$ ) content at baseline (prior to supplementation). (A) RBC $n$-3 DPA values in Study 1 explained $9.1 \%$ of the variability in triglyceride values $(y=139.3-2022 x)$; (B) In Study 2, RBC $n-3$ DPA values explained $11.4 \%$ of the variability in triglyceride values $(y=384-5922 x)$.

In Study 1, baseline RBC $n$-3 DPA content and serum CRP concentrations were also inversely related with a cubic fit $(p<0.001)$ (Figure 3A). This model fit suggests a threshold for effects, such that RBC $n$-3 DPA is inversely related to CRP at $n-3$ DPA values $<2 \%$ and/or higher CRP values. For $n-3$ DPA values $>2 \%$, there does not appear to be any predictive value of RBC $n-3$ DPA content with respect to CRP. There was no significant relationship between baseline RBC $n-3$ DPA and serum CRP in Study 2 (Figure 3B). No significant associations were found between RBC $n-3$ DPA content and the inflammatory cytokines IL-6 and TNF- $\alpha$ in Study 1 or Study 2 (data not shown).

(A) Study 1

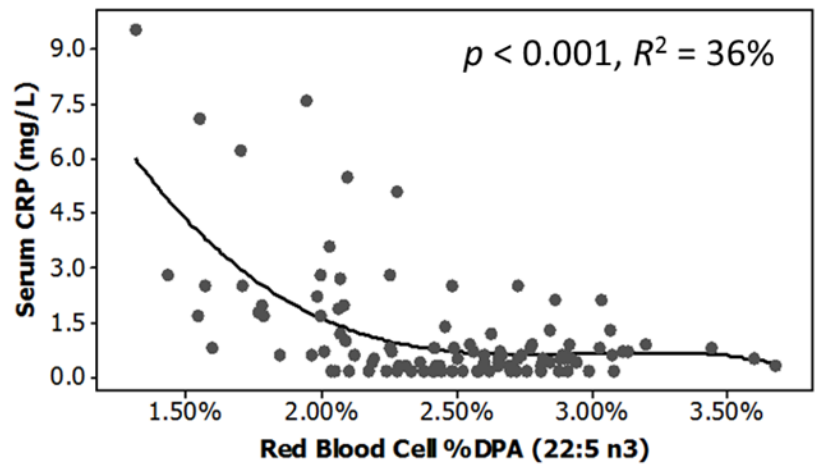

(B) Study 2

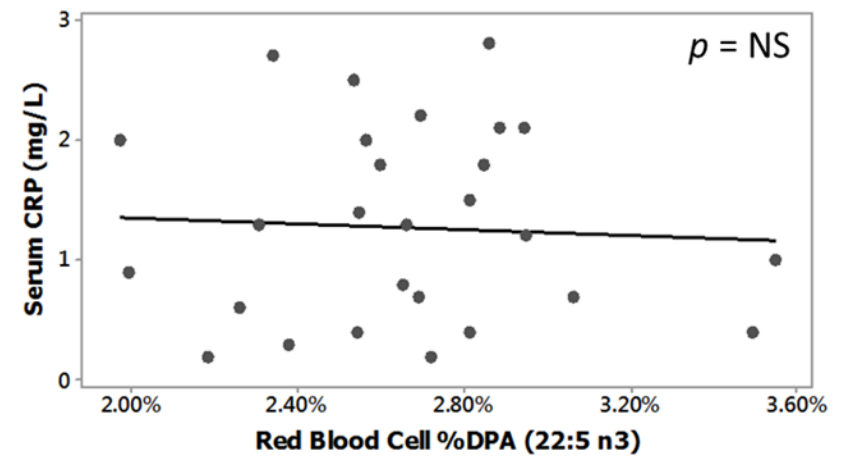

Figure 3. Scatterplots for regression analyses of serum C-reactive protein (CRP) vs. red blood cell (RBC) \% docosapentaenoic acid (DPA $n-3$ ) content at baseline (prior to supplementation). (A) RBC $n-3$ DPA values in Study 1 explained $36 \%$ of the variability in serum CRP using a cubic fit $\left(y=32.43-3236 x+109213 x^{2}-12221024 x^{3}\right)$; (B) In Study 2, RBC $n$-3 DPA values were not significantly related to serum CRP.

Sex differences in baseline (pre-supplementation) RBC $n-3$ DPA content were also found, with male participants having approximately $0.38 \%$ higher RBC $n-3$ DPA content compared to female participants (Figure 4). 


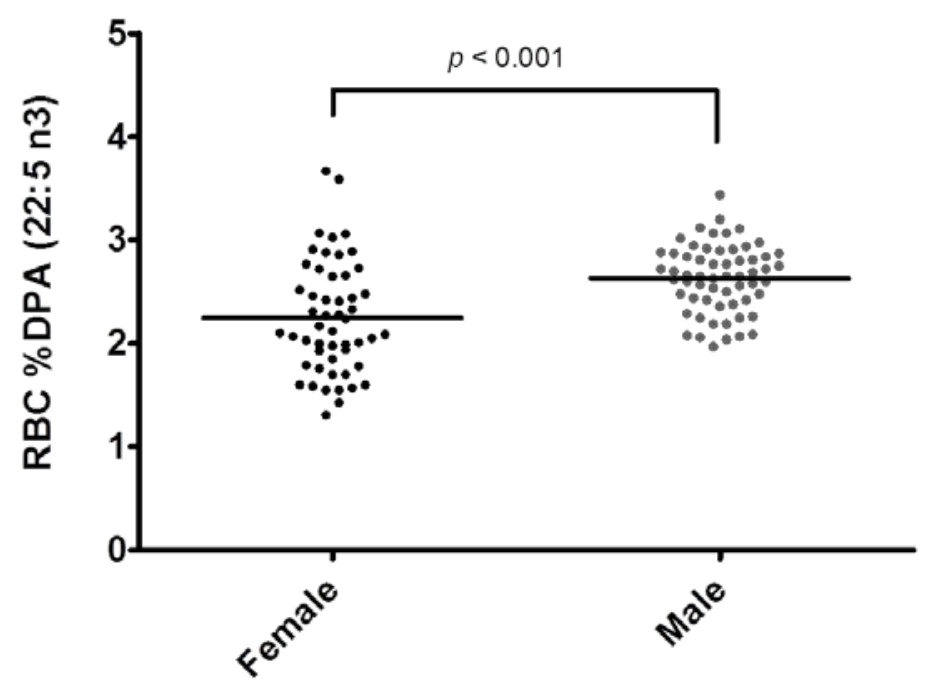

Figure 4. Sex differences in baseline red blood cell (RBC) \% docosapentaenoic acid (DPA $n-3)$ content.

\section{(A) Study 1}

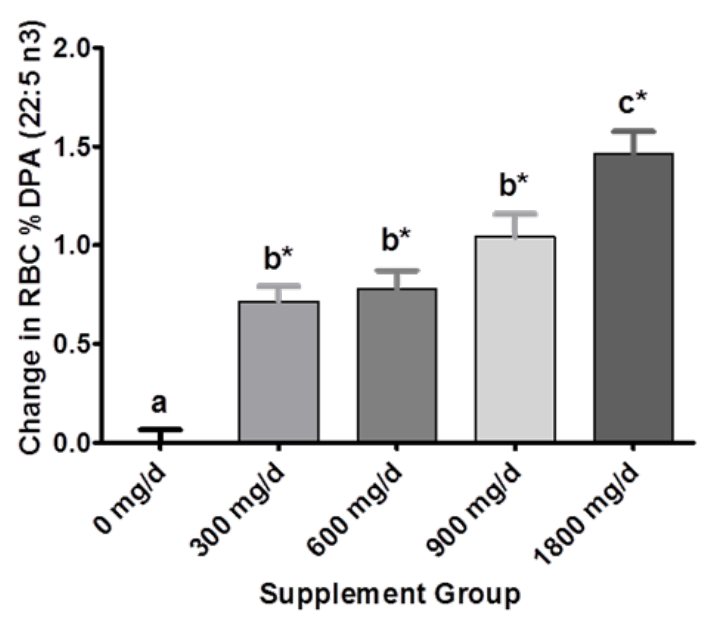

(B) Study 2

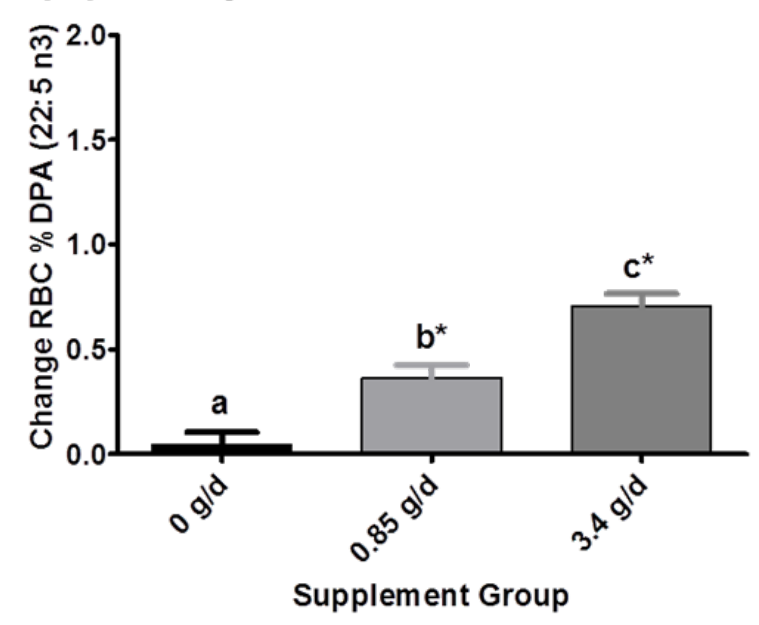

Figure 5. Mean (+/- standard error of the mean (SEM)) changes in red blood cell (RBC) docosapentaenoic acid (DPA $n-3$ ) content as a percentage of total fatty acids. Change scores for each treatment were calculated as end of treatment period value minus baseline value. Supplement groups refer to daily dose of Eicosapentaenoic acid (EPA) + docosahexaenoic acid (DHA) provided by one-gram oil capsules. (A) In Study 1, six capsules were consumed daily for five months; (B) In Study 2, four capsules were consumed daily for eight weeks. * Denotes significant difference vs. baseline. Different lowercase letters indicate significant differences between treatment groups, $p<0.05$ (Tukey-adjusted post hoc pairwise comparisons).

\subsection{Effects of Supplementation on RBC n-3 DPA Content}

Five months of supplementation with $1 \mathrm{~g}$ to $6 \mathrm{~g}$ fish oil capsules providing 300-1800 mg EPA + DHA (Study 1) dose-dependently increased the percent of $n$-3 DPA in RBCs (Figure 5A). A significant 29\% increase was observed for the lowest dose $(1 \mathrm{~g}$ standard fish body oil containing $300 \mathrm{mg}$ EPA + DHA 
and $20 \mathrm{mg} n$-3 DPA). The intermediate doses (600 mg/day and $900 \mathrm{mg} /$ day EPA + DHA) similarly increased RBC $n$-3 DPA content. Following the high dose (6 capsules/day, providing $1800 \mathrm{mg} /$ day $\mathrm{EPA}+\mathrm{DHA}$ and $120 \mathrm{mg} n-3 \mathrm{DPA}), \mathrm{RBC} n-3 \mathrm{DPA}$ content increased $61 \%$ relative to the placebo group. RBC $n$-3 DPA content also increased dose-dependently in Study 2, but to a lesser extent. A 14\% and 26\% increase in RBC $n$-3 DPA occurred when $850 \mathrm{mg} /$ day and $3400 \mathrm{mg} /$ day EPA + DHA were administered as prescription $n-3$ ethyl ester capsules provided for eight weeks (Figure 5B).

\section{Discussion}

To improve our understanding of the role of DPA in the health benefits derived from $n-3$ fatty acids, we performed secondary analyses of two published studies to examine the relationship between RBC n-3 DPA content, triglycerides, and inflammation, as well as the dose response effects of $n-3$ supplementation on RBC $n$-3 DPA stores. In healthy, younger adults, lower pre-supplementation RBC $n$-3 DPA concentrations were associated with higher levels of the inflammatory marker CRP. Lower RBC $n$-3 DPA was also associated with higher fasting triglycerides in these healthy adults and in individuals with moderately elevated triglycerides. Adjusting models for BMI, age, EPA, and DHA did not diminish the significance of RBC $n-3$ DPA as a predictor of CRP and triglycerides (data not shown). RBC EPA, $\mathrm{DHA}$, or EPA + DHA did not significantly predict CRP or fasting triglycerides in these studies. RBC $n-3$ DPA exhibited a dose-dependent increase following $n-3$ supplementation that was proportionally greater following five months of supplementation compared to eight weeks of supplementation.

The inverse relationship between RBC $n-3$ DPA and triglycerides that we observed has previously been documented in only one population [25], and may have implications for the management of atherogenic dyslipidemia. Given the well-documented triglyceride-lowering effect of EPA and DHA supplementation, it is surprising that only $\mathrm{RBC} n-3 \mathrm{DPA}$ was significantly related to fasting triglycerides in these two study populations and no relationship was found for EPA or DHA RBC content (both individually and in sum). We speculate that the lack of association may be related to the low habitual $n$-3 consumption of these participants, the conversion of dietary EPA to RBC $n-3$ DPA, and/or the influence of other factors that contribute to the development of high triglycerides. The efficacy of pure DPA supplementation for triglyceride reduction relative to EPA and DHA has not been studied. However, seal oil, a relatively rich source of DPA, has been shown to significantly reduce triglycerides in hypertriglyceridemic adults [26], with mixed results in healthy populations [27-30].

The inverse association between baseline RBC $n$-3 DPA content and serum CRP concentrations found in Study 1 agrees with prior studies [11-13,31,32] and may indicate a differential relationship between individual $n$-3 fatty acids and inflammatory markers. This inverse relationship with CRP has consistently been reported for plasma, serum, and RBC $n$-3 DPA concentrations [11-13,31,32]. In contrast, no association between CRP and RBC EPA or DHA content was found in the current study populations [4,23] or that of Labonte et al. [32]. Additionally, no relationship between RBC n-3 DPA content and inflammatory cytokines was found in either Study 1 or Study 2, despite there being an inverse relationship between RBC DHA and TNF- $\alpha$ in Study 1 [4]. EPA and DHA may promote resolution of inflammation via conversion into bioactive metabolites-resolvins, protectins, and maresins-collectively termed specialized pro-resolving mediators (SPMs). Novel $n$-3 DPA SPMs have also been characterized and demonstrated to have similar protective actions $[33,34]$. The 
anti-inflammatory effects of EPA may also in part be exerted via elongation to $n-3$ DPA as $n-3$ DPA produced from EPA supplementation was identified as the compound responsible for cyclooxygenase inhibition in vitro [35]. Based on the structural differences of $n$-3 DPA, EPA, DHA, and their metabolites, it is possible that they have unique effects in regulating different aspects of the innate immune system. The fatty acid composition of RBCs may also be differentially affected by the development of inflammation, and evaluating how RBC fatty acids are modified in the context of acute inflammation would serve to clarify this question.

The lack of association between RBC $n-3$ DPA and CRP in Study 2 may be attributed to several factors. In Study 2, the range of CRP values was much narrower (all baseline values were $<3 \mathrm{mg} / \mathrm{L}$ ). This study also had a much smaller sample size that consisted predominately of middle-aged Caucasian males and included only three post-menopausal women (pre-menopausal women were excluded due to the difficulty of scheduling endpoint assessments at the same phase in each menstrual cycle). In Study 1, females had higher CRP values at baseline [4] and had significantly less RBC $n$-3 DPA content compared to male participants (Figure 4). These factors may have largely driven the observed relationship between CRP and RBC $n$-3 DPA. Women of all age groups currently consume very little DPA (as well as EPA and DHA) [36,37], and prior studies have similarly found lower serum [38], plasma [39], and RBC n-3 DPA [40] concentrations in women. Future studies should further examine sex differences in DPA stores and whether these relate to levels of inflammation, as women are at higher risk of many inflammatory diseases.

Since $n$-3 DPA has been associated with inflammation [11-13,31,32] and several forms of cardiovascular disease [7,10,11,31], it is important to understand how $n-3$ DPA stores respond to $n-3$ supplementation. We found that RBC $n$-3 DPA content increased dose-dependently in both study populations. Significant increases were achieved even with the lowest dose (300 mg EPA + DHA), despite it providing only $20 \mathrm{mg} /$ day $n$-3 DPA. The greatest increase was achieved following 1800 mg/day EPA + DHA (118 mg/day $n$-3 DPA) for five months. Although RBC $n$-3 DPA content increased significantly in both studies, the relative magnitude of change was much smaller in Study 2, despite larger doses of $n-3$ being provided (i.e., 850 and $3400 \mathrm{mg} /$ day compared to 300, 600, 900, and $1800 \mathrm{mg} /$ day). This variance in RBC $n-3$ DPA responses may be due to differences in $n$-3 supplement preparation (i.e., triglyceride $v s$. ethyl ester forms), differences in participant characteristics, and/or the duration of supplementation. Earlier work has not demonstrated a significant difference in bioavailability between these preparations, and we did not observe the same discordance in RBC EPA responses between Study 1 and Study 2. Given that the average RBC lifespan is $\sim 120$ days, it is likely that the longer supplementation period of Study 1 (i.e., 20 weeks) allowed for greater RBC turnover, whereas in Study 2 , only a portion of the original RBCs were replaced with $n-3$ supplemented RBCs during the eight-week supplementation period. RBC $n$-3 DPA—like DHA [41] - may also be relatively more stable over time and require a longer period of supplementation or dietary change to achieve maximal levels compared to EPA [42].

As the amount of $n$-3 DPA contained in these supplements was small, it is likely that endogenous conversion of EPA to DPA via elongation by fatty acid elongase-2 (FAE-2) and fatty acid elongase-5 (FAE-5) occurred [43]. Prior clinical studies have also found increases in RBC and plasma $n-3$ DPA following supplementation with EPA + DHA [18,26,42] and purified EPA [19,20,44]. The baseline 
association between EPA and $n-3$ DPA - but not $n-3$ DPA and DHA - in our study populations and others similarly supports this [9,10,31]. This relationship between the long-chain $n-3$ fatty acids is also supported by cell-based studies in which inter-conversion of EPA and $n-3$ DPA occurs readily $[36,43,45]$, with limited conversion of $n-3$ DPA to DHA [46] and little evidence that DPA and DHA supplementation influence the production of one another $[19,47,48]$. Many have suggested that endogenous $n-3$ DPA stores are largely derived from the conversion of EPA based on poor correlations between plasma, serum, or RBC $n-3$ DPA and intake of fish or long-chain $n-3$ fatty acids of marine origin [9-11,31,32]. However, fish/seafood contains less $n$-3 DPA compared to EPA and DHA and more DPA is provided by ruminant meat sources [6,26,27]. Nutrient databases may also not currently provide adequate information about the DPA content of foods, similar to the lack of quantification of DPA content for most $n-3$ supplements. Although it has historically been difficult to obtain, pure DPA can be manufactured, and evaluating RBC fatty acid responses to pure EPA $v s$. pure DPA supplementation would help to clarify this relationship.

Furthermore, differences in the metabolism of the individual $n$ - 3 fatty acids and the inter-convertibility of n-3 DPA and EPA suggest that endogenous n-3 DPA reserves could serve as stores of EPA. In both cell-based [45] and clinical studies [47], DPA supplementation has been shown to increase EPA. There also appears to be cell/tissue specificity for $n$ - 3 storage as DHA is enriched in myocardial and neuronal membranes [49] and the EPA content of RBCs is lower than that of $n-3$ DPA and DHA [18,25,42,47]. It has been suggested that plasma EPA serves as a more dynamic and readily available pool of long-chain $n-3$ fatty acids that increases and decreases more quickly than DHA [10,41,42]. Thus, 22-carbon fatty acids may be preferentially stored in specific tissue compartments, and in the case of $n-3$ DPA, may serve to replenish plasma EPA that has been utilized. This may have important implications for the prevention or treatment of disease states known to be related to $n-3$ fatty acid intake and warrants further study.

Detailed descriptions of the respective strengths and limitations of Study 1 and Study 2 have previously been discussed [4,22,23]. For the context of the current analysis, both studies used well-characterized $n-3$ supplements, and compliance rates were very high. Participants for both studies were also recruited according to strict inclusion/exclusion criteria and were required to be low fish consumers. However, no other dietary intake data was collected. As red meat is a major dietary source of DPA, this could have influenced baseline RBC $n-3$ DPA concentrations, but it is unlikely that participants drastically changed their meat consumption over the course of the study. Both studies included men and women, but Study 2 included only three post-menopausal women. The sample size of both studies was also relatively small, and the majority of participants were generally healthy Caucasians, which may limit the generalizability of these results. These analyses were strengthened by the wide distribution of baseline fasting triglyceride concentrations in both Study 1 and Study 2. However, the limited range of CRP values in Study 2 may have limited our ability to observe a significant relationship with RBC n-3 DPA content in this population. The design of both studies allowed for the opportunity to evaluate dose-response effects and provided insight into potential duration-response effects of $n-3$ supplementation on RBC n-3 DPA content. Both studies also utilized RBC fatty acids, a recognized biomarker of long-term dietary $n-3$ intake. 


\section{Conclusions}

We have demonstrated that in both healthy adults and adults with moderately elevated triglycerides, RBC n-3 DPA content is associated with fasting triglycerides at baseline and dose-dependently increases in response to $n-3$ supplementation. Baseline CRP concentrations and RBC $n$-3 DPA were also inversely correlated in the population of healthy adults. Although this does not indicate causality, these results support the concept that $n$-3 DPA may play a role in the biological effects previously attributed solely to EPA and DHA. Further study is needed to replicate these findings in other populations and better characterize these relationships and the responsiveness of RBC $n-3$ DPA content to $n-3$ supplementation.

\section{Acknowledgments}

The authors would like to thank the research participants of both studies, as well as the nursing and clinician staff at the General Clinical Research Center of the Pennsylvania State University, which was supported by the National Center for Advancing Translational Sciences, National Institutes of Health, through Grant UL1TR000127. Study 1 was funded by the USDA and CSREES grant \#2009-65200-05973, and capsules were donated by Nordic Naturals. Financial support and capsules for Study 2 were provided by GlaxoSmithKline, and scholarship support from the National Fisheries Institute was awarded to Ann C. Skulas-Ray. Ann C. Skulas-Ray would also like to gratefully acknowledge travel support provided by Omega Protein that enabled presentation of the research at the 11th Congress of the International Society for the Study of Fatty Acids and Lipids.

\section{Author Contributions}

All authors were involved in the research conception and design; Michael R. Flock and Ann C. Skulas-Ray conducted the research and acquired data; William S. Harris performed the fatty acid analyses; Ann C. Skulas-Ray performed the statistical analysis and interpretation; and all authors were involved in the writing of the manuscript and take responsibility for the manuscript's final content.

\section{Conflicts of Interest}

Financial support had no role in the design and conduct of the study and in the collection, analysis, and interpretation of the data. William S. Harris is a member of the scientific advisory board of Aker Biomarine. He is the president of OmegaQuant Analytics, LLC (Sioux Falls, SD, USA), and is a senior research scientist at Health Diagnostic Laboratory, Inc. (Richmond, VA, USA), two companies that offer RBC omega-3 FA testing. Ann C. Skulas-Ray has received research support from Omega Protein and compensation for participation in a scientific advisory board meeting for AstraZeneca. None of the other authors have conflicts of interest to declare. 


\section{References}

1. Mozaffarian, D.; Wu, J.H. (n-3) fatty acids and cardiovascular health: Are effects of EPA and DHA shared or complementary? J. Nutr. 2012, 142, 614S-625S. [CrossRef] [PubMed]

2. Jacobson, T.A.; Glickstein, S.B.; Rowe, J.D.; Soni, P.N. Effects of eicosapentaenoic acid and docosahexaenoic acid on low-density lipoprotein cholesterol and other lipids: A review. J. Clin. Lipidol. 2012, 6, 5-18. [CrossRef] [PubMed]

3. Calder, P.C. $n-3$ fatty acids, inflammation and immunity: New mechanisms to explain old actions. Proc. Nutr. Soc. 2013, 72, 326-336. [CrossRef] [PubMed]

4. Flock, M.R.; Skulas-Ray, A.C.; Harris, W.S.; Gaugler, T.L.; Fleming, J.A.; Kris-Etherton, P.M. Effects of supplemental long-chain omega-3 fatty acids and erythrocyte membrane fatty acid content on circulating inflammatory markers in a randomized controlled trial of healthy adults. PLEFA 2014, 91, 161-168. [CrossRef] [PubMed]

5. Mozaffarian, D.; Wu, J.H. Omega-3 fatty acids and cardiovascular disease: Effects on risk factors, molecular pathways, and clinical events. J. Am. Coll. Cardiol. 2011, 58, 2047-2067. [CrossRef] [PubMed]

6. Howe, P.; Buckley, J.; Meyer, B. Long-chain omega-3 fatty acids in red meat. Nutr. Diet. 2007, 64, S135-S139. [CrossRef]

7. Rissanen, T.; Voutilainen, S.; Nyyssonen, K.; Lakka, T.A.; Salonen, J.T. Fish oil-derived fatty acids, docosahexaenoic acid and docosapentaenoic acid, and the risk of acute coronary events: The kuopio ischaemic heart disease risk factor study. Circulation 2000, 102, 2677-2679. [CrossRef] [PubMed]

8. Oda, E.; Hatada, K.; Katoh, K.; Kodama, M.; Nakamura, Y.; Aizawa, Y. A case-control pilot study on $n$-3 polyunsaturated fatty acid as a negative risk factor for myocardial infarction. Int. Heart J. 2005, 46, 583-591. [CrossRef] [PubMed]

9. Mozaffarian, D.; Lemaitre, R.N.; King, I.B.; Song, X.; Huang, H.; Sacks, F.M.; Rimm, E.B.; Wang, M.; Siscovick, D.S. Plasma phospholipid long-chain omega-3 fatty acids and total and cause-specific mortality in older adults: A cohort study. Ann. Intern. Med. 2013, 158, 515-525. [CrossRef] [PubMed]

10. Sun, Q.; Ma, J.; Campos, H.; Rexrode, K.M.; Albert, C.M.; Mozaffarian, D.; Hu, F.B. Blood concentrations of individual long-chain $n-3$ fatty acids and risk of nonfatal myocardial infarction. Am. J. Clin. Nutr. 2008, 88, 216-223. [PubMed]

11. De Oliveira Otto, M.C.; Wu, J.H.; Baylin, A.; Vaidya, D.; Rich, S.S.; Tsai, M.Y.; Jacobs, D.R.; Mozaffarian, D. Circulating and dietary omega-3 and omega-6 polyunsaturated fatty acids and incidence of CVD in the multi-ethnic study of atherosclerosis. J. Am. Heart Assoc. 2013, 2. [CrossRef] [PubMed]

12. Reinders, I.; Virtanen, J.K.; Brouwer, I.A.; Tuomainen, T.P. Association of serum n-3 polyunsaturated fatty acids with C-reactive protein in men. Eur. J. Clin. Nutr. 2012, 66, 736-741. [CrossRef] [PubMed] 
13. Micallef, M.A.; Munro, I.A.; Garg, M.L. An inverse relationship between plasma $n-3$ fatty acids and C-reactive protein in healthy individuals. Eur. J. Clin. Nutr. 2009, 63, 1154-1156. [CrossRef] [PubMed]

14. Sun, Q.; Ma, J.; Campos, H.; Hankinson, S.E.; Hu, F.B. Comparison between plasma and erythrocyte fatty acid content as biomarkers of fatty acid intake in us women. Am. J. Clin. Nutr. 2007, 86, 74-81. [PubMed]

15. Harris, W.S. Omega-3 fatty acids and cardiovascular disease: A case for omega-3 index as a new risk factor. Pharmacol. Res. 2007, 55, 217-223. [CrossRef] [PubMed]

16. Baylin, A.; Campos, H. The use of fatty acid biomarkers to reflect dietary intake. Curr. Opin. Lipidol. 2006, 17, 22-27. [CrossRef] [PubMed]

17. Harris, W.S. The omega-3 index: From biomarker to risk marker to risk factor. Curr. Atheroscler. Rep. 2009, 11, 411-417. [CrossRef] [PubMed]

18. Cao, J.; Schwichtenberg, K.A.; Hanson, N.Q.; Tsai, M.Y. Incorporation and clearance of omega-3 fatty acids in erythrocyte membranes and plasma phospholipids. Clin. Chem. 2006, 52, 2265-2272. [CrossRef] [PubMed]

19. Mori, T.A.; Burke, V.; Puddey, I.B.; Watts, G.F.; O’Neal, D.N.; Best, J.D.; Beilin, L.J. Purified eicosapentaenoic and docosahexaenoic acids have differential effects on serum lipids and lipoproteins, LDL particle size, glucose, and insulin in mildly hyperlipidemic men. Am. J. Clin. Nutr. 2000, 71, 1085-1094. [PubMed]

20. Von Schacky, C.; Weber, P.C. Metabolism and effects on platelet function of the purified eicosapentaenoic and docosahexaenoic acids in humans. J. Clin. Investig. 1985, 76, 2446-2450. [CrossRef] [PubMed]

21. Hill, A.M.; Buckley, J.D.; Murphy, K.J.; Howe, P.R. Combining fish-oil supplements with regular aerobic exercise improves body composition and cardiovascular disease risk factors. Am. J. Clin. Nutr. 2007, 85, 1267-1274. [PubMed]

22. Flock, M.R.; Skulas-Ray, A.C.; Harris, W.S.; Etherton, T.D.; Fleming, J.A.; Kris-Etherton, P.M. Determinants of erythrocyte omega-3 fatty acid content in response to fish oil supplementation: A dose response randomized controlled trial. J. Am. Heart. Assoc. 2013, 2, e000513. [CrossRef] [PubMed]

23. Skulas-Ray, A.C.; Kris-Etherton, P.M.; Harris, W.S.; Vanden Heuvel, J.P.; Wagner, P.R.; West, S.G. Dose-response effects of omega-3 fatty acids on triglycerides, inflammation, and endothelial function in healthy persons with moderate hypertriglyceridemia. Am. J. Clin. Nutr. 2011, 93, 243-252. [CrossRef] [PubMed]

24. Harris, W.S.; von Schacky, C. The omega-3 index: A new risk factor for death from coronary heart disease? Prev. Med. 2004, 39, 212-220. [CrossRef] [PubMed]

25. Dai, X.W.; Chen, Y.M.; Zeng, F.F.; Sun, L.L.; Chen, C.G.; Su, Y.X. Association between n-3 polyunsaturated fatty acids in erythrocytes and metabolic syndrome in Chinese men and women. Eur. J. Nutr. 2015. [CrossRef] [PubMed]

26. Meyer, B.J.; Lane, A.E.; Mann, N.J. Comparison of seal oil to tuna oil on plasma lipid levels and blood pressure in hypertriglyceridaemic subjects. Lipids 2009, 44, 827-835. [CrossRef] [PubMed] 
27. Mann, N.J.; O’Connell, S.L.; Baldwin, K.M.; Singh, I.; Meyer, B.J. Effects of seal oil and tuna-fish oil on platelet parameters and plasma lipid levels in healthy subjects. Lipids 2010, 45, 669-681. [CrossRef] [PubMed]

28. Deutch, B.; Jørgensen, E.B.; Hansen, J.C. $n$-3 PUFA from fish- or seal oil reduce atherogenic risk indicators in Danish women. Nutr. Res. 2000, 20, 1065-1077. [CrossRef]

29. Conquer, J.A.; Cheryk, L.A.; Chan, E.; Gentry, P.A.; Holub, B.J. Effect of supplementation with dietary seal oil on selected cardiovascular risk factors and hemostatic variables in healthy male subjects. Thromb. Res. 1999, 96, 239-250. [CrossRef]

30. Bonefeld-Jorgensen, E.C.; Moller, S.M.; Hansen, J.C. Modulation of atherosclerotic risk factors by seal oil: A preliminary assessment. Int. J. Circumpolar Health 2001, 60, 25-33. [PubMed]

31. Mozaffarian, D.; Lemaitre, R.N.; King, I.B.; Song, X.; Spiegelman, D.; Sacks, F.M.; Rimm, E.B.; Siscovick, D.S. Circulating long-chain omega-3 fatty acids and incidence of congestive heart failure in older adults: The cardiovascular health study: A cohort study. Ann. Intern. Med. 2011, 155, 160-170. [CrossRef] [PubMed]

32. Labonte, M.E.; Dewailly, E.; Lucas, M.; Couture, P.; Lamarche, B. Association of red blood cell $n-3$ polyunsaturated fatty acids with plasma inflammatory biomarkers among the Quebec Cree population. Eur. J. Clin. Nutr. 2014, 68, 1042-1047. [CrossRef] [PubMed]

33. Dangi, B.; Obeng, M.; Nauroth, J.M.; Teymourlouei, M.; Needham, M.; Raman, K.; Arterburn, L.M. Biogenic synthesis, purification, and chemical characterization of anti-inflammatory resolvins derived from docosapentaenoic acid (DPA n-6). J. Biol. Chem. 2009, 284, 14744-14759. [CrossRef] [PubMed]

34. Dalli, J.; Colas, R.A.; Serhan, C.N. Novel n-3 immunoresolvents: Structures and actions. Sci. Rep. 2013, 3, 1940. [CrossRef] [PubMed]

35. Norris, P.C.; Dennis, E.A. Omega-3 fatty acids cause dramatic changes in TLR4 and purinergic eicosanoid signaling. Proc. Natl. Acad. Sci. USA 2012, 109, 8517-8522. [CrossRef] [PubMed]

36. Ervin, R.B.; Wright, J.D.; Wang, C.Y.; Kennedy-Stephenson, J. Dietary Intake of Fats and Fatty Acids for the United States Population: 1999-2000; National Center for Health Statistics: Hyattsville, MD, USA, 2004; pp. 1-6.

37. U.S. Department of Agriculture, Agricultural Research Service. Total Nutrient Intakes: Percent Reporting and Mean Amounts of Selected Vitamins and Minerals from Food and Dietary Supplements. 2012. Available online: www.ars.usda.gov/ba/bhnrc/fsr (accessed on 29 May 2015).

38. Crowe, F.L.; Skeaff, C.M.; Green, T.J.; Gray, A.R. Serum n-3 long-chain PUFA differ by sex and age in a population-based survey of New Zealand adolescents and adults. Br. J. Nutr. 2008, 99, 168-174. [CrossRef] [PubMed]

39. Garneau, V.; Rudkowska, I.; Paradis, A.M.; Godin, G.; Julien, P.; Perusse, L.; Vohl, M.C. Omega-3 fatty acids status in human subjects estimated using a food frequency questionnaire and plasma phospholipids levels. Nutr. J. 2012, 11, 46. [CrossRef] [PubMed]

40. Howe, P.R.; Buckley, J.D.; Murphy, K.J.; Pettman, T.; Milte, C.; Coates, A.M. Relationship between erythrocyte omega-3 content and obesity is gender dependent. Nutrients 2014, 6, 1850-1860. [CrossRef] [PubMed] 
41. Brown, A.J.; Pang, E.; Roberts, D.C. Persistent changes in the fatty acid composition of erythrocyte membranes after moderate intake of $n-3$ polyunsaturated fatty acids: Study design implications. Am. J. Clin. Nutr. 1991, 54, 668-673. [PubMed]

42. Katan, M.B.; Deslypere, J.P.; van Birgelen, A.P.; Penders, M.; Zegwaard, M. Kinetics of the incorporation of dietary fatty acids into serum cholesteryl esters, erythrocyte membranes, and adipose tissue: An 18-month controlled study. J. Lipid Res. 1997, 38, 2012-2022. [PubMed]

43. Kaur, G.; Cameron-Smith, D.; Garg, M.; Sinclair, A.J. Docosapentaenoic acid (22:5 n-3): A review of its biological effects. Prog. Lipid Res. 2011, 50, 28-34. [CrossRef] [PubMed]

44. Krul, E.S.; Lemke, S.L.; Mukherjea, R.; Taylor, M.L.; Goldstein, D.A.; Su, H.; Liu, P.; Lawless, A.; Harris, W.S.; Maki, K.C. Effects of duration of treatment and dosage of eicosapentaenoic acid and stearidonic acid on red blood cell eicosapentaenoic acid content. PLEFA 2012, 86, 51-59. [CrossRef] [PubMed]

45. Benistant, C.; Achard, F.; Ben Slama, S.; Lagarde, M. Docosapentaenoic acid (22:5 n-3): Metabolism and effect on prostacyclin production in endothelial cells. PLEFA 1996, 55, 287-292. [CrossRef]

46. Kaur, G.; Begg, D.P.; Barr, D.; Garg, M.; Cameron-Smith, D.; Sinclair, A.J. Short-term docosapentaenoic acid (22:5 n-3) supplementation increases tissue docosapentaenoic acid, DHA and EPA concentrations in rats. Br. J. Nutr. 2010, 103, 32-37. [CrossRef] [PubMed]

47. Miller, E.; Kaur, G.; Larsen, A.; Loh, S.P.; Linderborg, K.; Weisinger, H.S.; Turchini, G.M.; Cameron-Smith, D.; Sinclair, A.J. A short-term n-3 DPA supplementation study in humans. Eur. J. Nutr. 2013, 52, 895-904. [CrossRef] [PubMed]

48. Linderborg, K.M.; Kaur, G.; Miller, E.; Meikle, P.J.; Larsen, A.E.; Weir, J.M.; Nuora, A.; Barlow, C.K.; Kallio, H.P.; Cameron-Smith, D.; et al. Postprandial metabolism of docosapentaenoic acid (DPA, 22:5 n-3) and eicosapentaenoic acid (EPA, 20:5 n-3) in humans. PLEFA 2013, 88, 313-319. [CrossRef] [PubMed]

49. Harris, W.S.; Sands, S.A.; Windsor, S.L.; Ali, H.A.; Stevens, T.L.; Magalski, A.; Porter, C.B.; Borkon, A.M. Omega-3 fatty acids in cardiac biopsies from heart transplantation patients: Correlation with erythrocytes and response to supplementation. Circulation 2004, 110, 1645-1649. [CrossRef] [PubMed]

(C) 2015 by the authors; licensee MDPI, Basel, Switzerland. This article is an open access article distributed under the terms and conditions of the Creative Commons Attribution license (http://creativecommons.org/licenses/by/4.0/). 